\title{
Diodoro Sículo y los reyes de Roma
}

\author{
Jorge MARTíneZ-PinNA \\ Universidad de Málaga \\ jmn@uma.es
}

\section{RESUMEN}

En este artículo se analizan los fragmentos de Diodoro Sículo relativos a los reyes de Roma. A pesar de su escaso contenido, se puede suponer que Diodoro se aleja de la tradición representada por Livio y se aproxima más a Dionisio de Halicarnaso y en parte a Cicerón.

Palabras clave: Diodoro Sículo, Roma, monarquía.

\section{Diodore de Sicile et des rois de Rome}

\section{RÉSUMÉE}

Cet article analyse les fragments de Diodore de Sicile sur les rois de Rome. Malgré leur insuffisante information, on peut suposer que Diodore a utilisé des sources différentes à la tradition représentée par Tite-Live et plus prochaines à celles de Denys d'Halicarnasse et aussi de Cicéron.

Mots clé: Diodore de Sicile, Rome, royauté.

Dino Ambaglio in memoriam

La historia de la Roma arcaica representa una verdadera crux en los estudios sobre Diodoro Sículo, tanto por el planteamiento que ofrece de la misma como por las fuentes que utiliza. Las opiniones de los modernos al respecto son muy variadas, señalando todas las direcciones posibles. En honor a la verdad, forzoso es reconocer que en su mayoría no carecen de fundamento. Incluso la primera de ellas, aquélla que señalaba a Fabio Píctor como fuente principal de Diodoro ${ }^{1}$, no deja de ser en parte acep-

1 Th. Mommsen, «Fabius und Diodor», Hermes, 13, 1878, 305-330, quien desarrolla una sugerencia

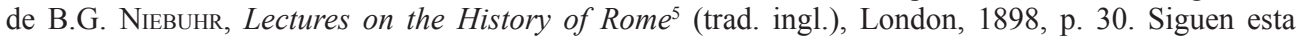
corriente, entre otros, E. Schwarz, «Diodoros von Agyrion», RE, V, 1903, col. 696 ss.; O. Leuze, Die römische Jahrzählung, Tübingen, 1909, pp. 69 ss.; F. CoRnelius, Untersuchungen zur frühen römischen Geschichte, München, 1940, p. 34. 
table, a pesar del rechazo general que ha experimentado en los últimos tiempos ${ }^{2}$. Hay que tener presente que Fabio es el único autor antiguo que Diodoro menciona por su nombre en su exposición sobre la Roma primitiva y arcaica, si bien él prefiere seguir otra versión en el asunto que trata, el origen del topónimo $\mathrm{Alba}^{3}$. Pero este rechazo de Fabio en un punto concreto no implica necesariamente que Diodoro no conociese la obra del historiador romano, o que sólo le utilizase para decir lo opuesto. Más bien al contrario, no creo que pueda ponerse en duda que Diodoro disponía de los anales de Fabio, cuya obra circulaba por Sicilia ya en el siglo II a.C. ${ }^{4}$, y que por el hecho de estar redactados en lengua griega, suponía para Diodoro una facilidad añadida. Sin embargo, si todas estas opiniones pueden ser en general al menos parcialmente aceptables, también hay que reconocer que ninguna de ellas resulta por completo satisfactoria. De ahí que no falte quien en vez de buscar un autor o autores concretos, se inclinen por tendencias, como sugiere S. Mazzarino al hablar de una tradición "fabia" y otra "claudia", presente esta última con mayor fuerza en la obra de Diodoro5. Pero aun así, la conclusión última no mejora sustancialmente respecto a lo anterior. Parece probado que Diodoro utilizó, especialmente en su exposición sobre la Roma altorepublicana, una fuente narrativa y otra cronográfica ${ }^{6}$, y que esta última ofrece la mejor de las versiones ${ }^{7}$, pero por el momento resulta imposible inclinarse con seguridad hacia una dirección determinada. En suma, quizá lo más acertado sería asumir, en palabras de G. Poma, que «la fonte (o le fonti) di Diodoro sono destinate forse a

2 En contra de la teoría "fabiana" se decantan A. SchwegLer, Römische Geschichte, Tübingen, 1856, vol. II.1, pp. 23 s.; B. NIESE, «Die Chronologie der gallischen Kriege bei Polybios», Hermes, 13, 1878, p. 412; E. MEYER, «Untersuchungen über Diodor's römische Geschichte», RhM, 37, 1882, pp. 610 ss.; L. Cohn, «Diodor und seine römische Quelle», Philologus, 42, 1884, pp. 9 ss.; E. Kornemann, Der Priestercodex in der Regia und die Entstehung der altrömischen Pseudogeschichte, Tübingen, 1912, pp. 32 s.; K.J. Beцoch, Römische Geschichte, Berlin, 1926, pp. 126 ss. Este último se inclina por una analista de época silana, quizá Claudio Quadrigario, opinión que siguen, en sus líneas generales, A. KLotz, «Diodors römische Annalen», RhM, 86, 1937, pp. 206 s.; IDEM, Livius und seine Vorgänger, Stuttgart, 1941, vol. III, p. 273 (Klotz rechaza que pueda ser Claudio); J. WolsKI, «La prise de Rome par les Celtes et la formation de l'annalistique romaine», Historia, 5, 1956, p. 25. Por su parte, niega una derivación de Fabio en beneficio de C. Acilio, F. Altheim, «Diodors römische Annalen», RhM, 93, 1950, esp. p. 285. Más distante se muestra E. MANNI, «Diodoro e la storia arcaica di Roma», Kokalos,

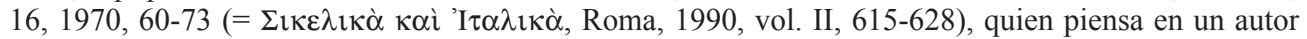
griego como Sileno o Filino.

${ }^{3}$ Diod., 7.5.4-5; Fab. Pict., fr. 4 P = fr. 5 Ch. Véase recientemente G. Vanotti, «Diodoro epitomato

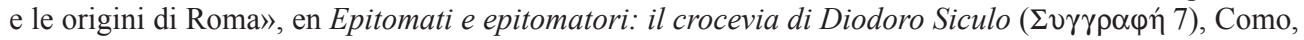
2005 , pp. 222 ss.

${ }^{4}$ Así parece mostrarlo la inscripción histórica, fechada en el siglo II y con el nombre de Fabio Píctor, procedente del gimnasio de Tauromenion: Fabio, fr. $1 \mathrm{Ch}$; G. MAnganaro, «Una biblioteca storica del ginnasio di Tauromenion e il P.Oxyr. 1245», PdP, 29, 1974, pp. 395 ss.; IDEM, en A. Alföldi, Römische Frühgeschichte, Heidelberg, 1976, pp. 87 ss.

${ }^{5}$ S. Mazzarino, Il pensiero storico classico, Bari, 1994, vol. II, pp. 293 ss.

${ }^{6}$ K.J. Beloch, Römische Geschichte, pp. 107 ss.

${ }^{7}$ F. CAsSola, «Diodoro e la storia romana», $A N R W$, II.30.1, 1982, pp. 728 ss. 
restare inafferrabili $»^{8}$, o todo lo más, como dice F. Cassola, «accontentarsi di definire alcune caratteristiche generali della fonte, senza proporne una identificazione» ${ }^{9}$.

Existe una impresión generalizada entre los autores modernos que Diodoro trató con mayor amplitud la historia de la Roma monárquica que la altorepublicana, y en efecto así parece. Aunque sólo se conozcan a través de resúmenes, los libros VII-X de Diodoro incluían el relato de la llegada de Eneas a Italia, de la dinastía albana, de la fundación de Roma y de los diferentes reyes romanos. Sin embargo, inmediatamente después de narrar la caída del régimen monárquico, Diodoro sólo consignaba los cónsules del año y en ocasiones escasas referencias a los acontecimientos del mismo. ¿A qué puede obedecer tal actitud? En opinión de G. Poma, esta diferencia en el tratamiento histórico de Roma no se justifica tanto por una decisión personal de Diodoro, de acuerdo con sus gustos e intereses, sino sobre todo por la dependencia que manifiesta respecto a su fuente principa ${ }^{10}$. Pero tal explicación no se acepta con comodidad. Aunque algunos historiadores romanos incidiesen con mayor profundidad en la historia de la monarquía que en los primeros tiempos republicanos, la exigüidad de las noticias de Diodoro para el siglo V no se corresponde con el tratamiento que, en referencia a esta época, se pudiese encontrar en el analista más conciso. Diodoro dispone de hecho de las mismas fuentes que Dionisio de Halicarnaso -siempre que demos por buena su afirmación de conocer los documentos oficiales romanos ${ }^{11}-$, pero el resultado entre uno y otro no puede ser más distante en lo que a la República se refiere.

Según creo, la respuesta debe encaminarse hacia los propios planteamientos e intereses de Diodoro como historiador. Su obra es una historia universal, pero redactada desde una perspectiva griega. Hasta el momento en que Roma no surge como potencia y despierta la atención de los griegos, es decir con las guerras samnitas como muy pronto, su historia carece prácticamente de interés. No deja de ser un mero apéndice. La existencia de Roma es continuamente recordada, mediante la mención de sus magistrados epónimos, como un anuncio de su grandeza futura al estar destinada a convertirse en señora del mundo. La importancia secundaria que en la obra de Diodoro presenta Roma queda patente en el número de capítulos que le dedica en los libros I-XXII, apenas un $3 \%$ en relación a los consagrados al mundo griego, incluida Sicilia $^{12}$. Si para la época arcaica los avatares de un Estado en formación, como era Roma, podían suscitar cierta curiosidad, a partir del siglo V los hechos griegos, tanto en la Grecia propia como en Sicilia y Magna Grecia, alcanzaron una importancia muy por encima del interés que pudiera despertar la historia romana. El protagonismo tiene que corresponder necesariamente al mundo griego. Los acontecimientos de Roma,

8 G. Poma, Tra legislatori e tiranni, Bologna, 1984, p. 107. En similar sentido G. Sigwart, «Römische Fasten und Annalen bei Diodor», Klio, 6, 1906, p. 378: "Wer der Historiker war, der Diodor als unmittelbare Vorlage diente, ist schwer zu sagen".

9 F. CASSOLA, «Diodoro e la storia romana», p. 752.

${ }^{10}$ G. Poma, Tra legislatori e tiranni, p. 113.

11 Diod., 1.4.4. Cf.; S. Mazzarino, Il pensiero storico classico, vol. II, p. 294; M. Rusconi, «Le notizie romane di Diodoro e gli 'Annales maximi'», en Storiografia e porpaganda (CISA 3), Milano, 1975, pp. 105 s. De hecho, Diodoro vivió en Roma durante más de 20 años: K.S. SACKs, Diodorus Siculus and the First Century, Princeton, 1990, p. 126.

${ }^{12}$ Cf. K.S. SACKS, Diodorus Siculus and the First Century, p. 118, n. 2. 
marcados por una continua monotonía tanto en el exterior como en el interior, apenas suscitan la atención de un lector extraño a los mismos. Sólo algunos hechos de cierta trascendencia (creación del tribunado, decenvirato, catástrofe gala) merecerían ser reseñados con cierto detalle. Y aun así Diodoro parece inclinarse en ocasiones por la descripción de aspectos secundarios, o incluso superfluos, pero más entretenidos, en vez de centrarse en el núcleo del acontecimiento, como se observa por ejemplo a propósito del decenvirato ${ }^{13}$. En cierto sentido, Diodoro regresa a la situación respecto a Roma que ya existía en la historiografía greco-occidental del siglo IV, que como ha mostrado E. Gabba ${ }^{14}$, sentía un mayor interés hacia la monarquía, sin preocuparse apenas por la historia republicana.

En realidad sabemos muy poco sobre cómo trataba Diodoro la historia de la época monárquica romana. Su testimonio ha llegado muy fragmentado, en su mayor parte en forma de resúmenes englobados en la colección bizantina conocida como Excerpta Constatiniana, redactada en el siglo X a iniciativa del emperador Constatino VII Porfirogéneta ${ }^{15}$. Pero los intereses de los autores que realizaron la selección eran muy diferentes a los de un moderno, y en consecuencia el resultado dista mucho de satisfacer nuestras necesidades. No obstante, para la etapa más antigua, aquella relativa a la presencia de Eneas en el Lacio y la dinastía albana, disponemos también de la crónica de Eusebio, lo cual incrementa notablemente nuestro caudal de información. A partir de estos datos, F. Cassola ha podido ofrecer un extenso y brillante estudio sobre las fuentes utilizadas por Diodoro, con conclusiones muy lejanas respecto a lo dicho con anterioridad ${ }^{16}$. La idea de que Diodoro utiliza una única fuente para un período determinado, no parece que pueda aplicarse a la historia de los orígenes de Roma. En el estudio de Cassola, se señalan como elementos más destacados la utilización de Varrón para el relato sobre Eneas y una notable coincidencia con Dionisio de Halicarnaso en lo relativo a la dinastía albana, lo que hace suponer que ambos derivarían de la misma fuente, probablemente los anales de los pontífices.

El objeto del presente trabajo no es otro que aquellos fragmentos, pocos, que interesan a la narración sobre los reyes de Roma y que fueron excluidos en el estudio de Cassola. Se trata de leves referencias a las personas, más que a los reinados, de Numa Pompilio, Tulo Hostilio, Tarquinio Prisco y Servio Tulio (Diod., 8.14; 8.25-26; 8.31; 10.1-2). Según comprobaremos en las páginas que siguen, los datos a nuestra disposición son muy escasos, lo cual limita notablemente las posibilidades de interpretación. No se espere entonces encontrar aquí una respuesta satisfactoria a las múltiples cues-

13 Diod., 12.24-25. Cf. G. Poma, Tra legislatori e tiranni, p. 119: "Per i decemviri, in Diodoro c'è solo silenzio, o poco più".

14 E. GABBA, «Considerazioni sulla tradizione letteraria sulle origini della Repubblica», en Les origines de la République romaine, Vandoeuvres, 1967, pp. 165 ss. (= Roma arcaica. Storia e storiografia, Roma, 2000, pp. 46 s.).

15 Recientemente, B. Flusin, «Les Excerpta constatiniens: logique d'une anti-histoire», en Fragments d'historiens grecs. Autour de Denys d'Halicarnasse, Roma, 2002, 537-559; M. CASEvitz, «Sur les fragments d'historiens grecs, particulièrement Diodore de Sicile», ibidem, 449-460.

16 F. CASSOLA, «Le origini di Roma e l'età regia in Diodoro», en Mito, storia, tradizione. Diodoro Siculo e la storiografia classica, Catania, 1991, 273-324. Con posterioridad a este estudio, han incidido sobre el mismo asunto G. VAnotTI, «Diodoro epitomato e le origini di Roma», cit., y R. SAMmartano, «La leggenda troiana in Diodoro», en Diodoro Siculo e la Sicilia indigena, Palermo, 2006, 10-25. 
tiones que plantea el texto de Diodoro. Mi objetivo, más bien modesto, es tan sólo incidir sobre algunos aspectos que puedan servir de base para una futura discusión.

\section{Diodoro, 8.14: Numa y Pitágoras}

El episodio de la formación pitagórica del rey romano Numa Pompilio es recordado también, con mayor o menor detalle, por otros autores, destacando al respecto Cicerón, Livio, Dionisio de Halicarnaso y Plutarco ${ }^{17}$. Los tres primeros rechazan abiertamente la historicidad de esta tradición basándose sobre todo en consideraciones cronológicas, ya que en efecto entre Numa y Pitágoras media un largo espacio de tiempo. Sin embargo, tampoco se aprecia entre todos ellos una absoluta coincidencia sobre cuándo llegó Pitágoras a Italia: Livio y Dionisio parecen situar este hecho durante el reinado de Servio Tulio, mientras que Cicerón, quien utiliza un cómputo cronológico griego ${ }^{18}$, lo retrasa a la época de Tarquinio el Soberbio, situando la llegada de Pitágoras a Italia en el cuarto año de su reinado. Plutarco adopta por su parte una posición menos comprometida. En el primer capítulo de su biografía sobre Numa, recoge diversas versiones que en parte concuerdan con las anteriores en una valoración negativa: así, al igual que Livio, recuerda que Numa alcanzaría la virtud por sus propios medios, o que entre Numa y Pitágoras habría un lapso de cinco generaciones (cuatro según Dionisio); pero deja la puerta abierta a una solución de compromiso al sugerir que pudiera tratarse de otro Pitágoras, en este caso un espartano contemporáneo de Numa, aduciendo como pruebas por una parte la similitud de costumbres entre Esparta y Roma, y por otra la tradición sobre el origen lacedemonio de los sabinos ${ }^{19}$. Pero más adelante, Plutarco resalta los elementos de contacto entre algunas disposiciones religiosas de Numa y las doctrinas de los pitagóricos, señalando además aquellos aspectos que introducen a Pitágoras en el mundo romano, como su supuesta ciudadanía y la estatua que se le erigió en el Foro ${ }^{20}$.

La tradición sobre la presencia de Numa en la escuela de Pitágoras es muy antigua. Recientemente M. Humm ha retomado el "dossier", concluyendo que la leyenda nació en ambiente romano en la segunda mitad del siglo IV, coincidiendo con los mo-

${ }^{17}$ Cic., Rep., 2.15.28-29; Liv., 1.18.2-4; Dion., 2.59; Plut., Num., 1.3-5, 8.5-21.

18 Cicerón sigue bien el liber annalis de su amigo T. Pomponio Atico (F. MünZER, «Atticus als Geschichtsschreiber», Hermes, 40, 1905, p. 81) o bien a Polibio (cf. F.W. Walbank, A Historical Commentary on Polybius, Oxford, 1970, vol. I, p. 666). La coincidencia entre Pitágoras y Tarquinio el Soberbio es también señalada por Aulo Gelio (Noct. At., 17.21.6), quien posiblemente deriva de Cornelio Nepote, amigo de Cicerón y de Atico.

19 Plut., Num., 1.3-7. El origen espartano de los sabinos era ya mencionado por Catón, fr. $51 \mathrm{P}$ = fr. II.22 Ch (= Serv. auct., Aen., 8.638). Sobre el particular, J. PoucET, «Les origines mythiques des Sabins», en Études Étrusco-Italiques, Louvain, 1963, 155-225; C. LetTA, «I mores dei Romani e l'origine dei Sabini in Catone», en Presitoria, storia e civiltà dei Sabini, Rieti, 1985, 15-34; D. BRIQueL, «La tradizione letteraria sull'origine dei Sabini», en Identità e civiltà dei Sabini, Firenze, 1996, 29-40; J. Martínez-Pinna, La prehistoria mítica de Roma, Madrid, 2002, pp. 60 ss.

20 Plut., Num., 8.5-2; a la estatua se refiere también Plin., Nat. hist., 34.26. Véase M. SeHLMeYer, Stadtrömische Ehrenstatuen der republikanischen Zeit, Stuttgart, 1999, pp. 88 ss. 
mentos de mayor influencia del pitagorismo sobre la nobilitas romana ${ }^{21}$. El punto de partida habría que localizarlo en Tarento, hacia donde E. Gabba ya dirigió la mirada, y más en concreto al filósofo Aristoxeno, como posible origen del mito ${ }^{22}$. Sea como fuere, es indudable que esta tradición gozó de una amplia aceptación en Roma desde comienzos del siglo II, pues sólo así se justifica el célebre episodio de los libros de Numa en el año $181^{23}$, pero ya a mediados del siglo I a.C. había perdido gran parte de su fuerza, como queda patente en los mencionados pasajes de Cicerón, Livio y Dionisio.

¿Cuál era la posición de Diodoro? El fragmento es demasiado breve para conocer con certidumbre hacia dónde se inclinaban sus preferencias. La alusión al vínculo entre Numa y Pitágoras se introduce con un $\lambda \dot{\varepsilon} \gamma 0 v \sigma \imath$ $\delta \varepsilon$ $\tau \imath \varepsilon \varepsilon \varsigma$ que indica que Diodoro se hace eco de una versión muy extendida. Si sus ideas estuviesen por completo resumidas en el fragmento transmitido, habría que reconocer entonces que sigue una fuente del siglo II, según la opinión común, antes de que esta tradición entrase en completa decadencia. De esta forma, Th. Mommsen pensó en Fabio Píctor, quien probablemente también conocería la leyenda ${ }^{24}$. Sin embargo, Diodoro vuelve a referirse a Pitágoras en otro lugar ${ }^{25}$, situando la acmé del filósofo en la olimpiada 61, coincidiendo, según la interpretación de R. Werner, con la entronización en Roma de Tarquinio el Soberbio ${ }^{26}$, con lo cual se aproxima a la cronología de Cicerón. A partir de estos datos, hemos de suponer por tanto que Diodoro rechazaba también la validez histórica de la tradición que vinculaba a Numa y Pitágoras.

${ }^{21}$ M. Humm, «Les origines du pythagorisme romain: problèmes historiques et philosophiques. I», LEC, 64, 1996, pp. 340 ss.; IDEM, «Numa et Pythagore: vie et mort d'un mythe», en Images d'origines. Origines d'une image, Louvain-la-Neuve, 2004, 125-137; IDEM, Appius Claudius Caecus. La République accomplie, Roma, 2005, pp. 547 ss. Recientemente, F. Russo, Pitagorismo e spartanità. Elementi politico-culturali tra Taranto, Roma ed i Sanniti alla fine del IV sec. a.C., Campobasso, 2007, pp. 58 ss., pone él énfasis en la gens Aemilia come creadora y difusora de laleyenda a finales del siglo IV.

${ }^{22}$ E. GABBA, «Considerazioni sulla tradizione letteraria sulle origini della Repubblica», pp. 155 s. (= Roma arcaica, pp. 39 ss.). Esta cronología es aceptada de manera casi general: P. WuILLEUMIER, Tarente des origines à la conquête romaine, Paris, 1939, p. 665; L. FERRERO, Storia del pitagorismo nel mondo romano dalle origini alla fine della Repubblica, Torino, 1955, p. 143; R.M. OGILVIE, A Commentary on Livy. 1-5, Oxford, 1965, p. 89; A. StorChi Marino, Numa e Pitagora, Napoli, 1999, pp. 50 s. En contra, P. PAnitscheK, «Numa Pompilius als Schüler des Pythagoras», GB, 17, 1990, 25-42, quien se inclina por la analística del siglo II a.C.

23 Liv., 40.29; Plin., Nat. hist., 13.27; Plut., Num., 22; Val. Max., 1.1.12; Fest., 178L; Auct. vir. ill., 3.2; Lact., Inst., 1.22; Aug., Civ. Dei, 7.34. Véanse A. Delatte, «Les doctrines pythagoriciennes des livres de Numa», $B A B, 22,1936,19-40$; K.R. Prowse, «Numa and the Pythagoreans: a curious incident», G\&R, 2, 1964, 36-42; M.J. PenA, «La tumba y los libros de Numa», Faventia, 1, 1979, 213-229; A. Willi, «Numa's Dangerous Books», MH, 55, 1998, 139-172; A. Storchi Marino, Numa e Pitagora, pp. 163 ss.

24 Th. Mommsen, «Fabius und Diodor», p. 315. Así también A. Rosenberg, Einleitung und Quellenkunde zur römischen Geschichte, Berlin, 1921, pp. 117 s. (Fabio y sus seguidores inmediatos que escribían en griego); L. Ferrero, Storia del pitagorismo nel mondo romano, p. 145.

25 Diod., 10.3.

26 R. Werner, Der Beginn der römischen Republik, Wien, 1963, p. 169. Según R.T. RIDLEY, «The Enigma of Servius Tullius», Klio, 57, 1975, pp. 154 s., Diodoro hace contemporáneos a Pitágoras y Servio Tulio, por lo que derivaría de un anticuario de la baja República. 
No obstante, se observa en el texto un detalle que no carece de interés: Numa fue llamado a ocupar el trono de Roma por su educación pitagórica, impresión en la que coincide con Dionisio, y fue asimismo de Pitágoras de quien recibió las normas para regular el culto. Teniendo además en cuenta que cuando trata más ampliamente sobre Pitágoras en el tercer capítulo del libro X, Diodoro alaba la personalidad y doctrinas del filósofo, no sería aventurado creer que en última instancia derivase de un autor, preferentemente griego, conocedor de las doctrinas pitagóricas, pero que negaba que Numa y Pitágoras fuesen contemporáneos. Dionisio llegaba a esta conclusión tras realizar una investigación propia, pero no parece que Diodoro actuara de similar manera, sino que ya debió encontrar en su fuente la falta de sincronía entre ambos personajes.

A título de hipótesis, podría proponerse a Cástor de Rodas como fuente última de Diodoro para este episodio concreto. No se puede ocultar que con este nombre entramos en un terreno peligroso. Como se sabe, existía una antigua tendencia a ver en Cástor la fuente cronográfica de Diodoro ${ }^{27}$, pero en la actualidad ha sido por completo descartada y no sin buenos argumentos ${ }^{28}$. Sin embargo, esto no implica necesariamente que Cástor fuese por completo desconocido por Diodoro ${ }^{29}$, o que más probablemente pudiera derivar de él a través de un autor interpuesto. Si se acepta esta última posibilidad, resulta inevitable pensar en Varrón, quien no se vio al margen de la influencia de Cástor ${ }^{30}$ y que a su vez fue utilizado tanto por Diodoro como por Dionisio de Halicarnaso para las fases más antiguas de Roma. Como se sabe por Plutarco, el historiador rodio buscaba trazar un vínculo entre las costumbres romanas y las prácticas pitagóricas ${ }^{31}$, por lo que no le sería desconocida la tradición de Numa y Pitágoras, que él interpretaría en clave favorable al filósofo griego. Así, no en vano se le ha señalado como probable fuente de Plutarco en la exposición que éste ofrece sobre las relaciones doctrinales entre ambos personajes ${ }^{32}$. Esta hipótesis se refuerza por el hecho de que Cástor trató asimismo sobre la Roma más antigua, disertando sobre los reyes de Alba y de Roma ${ }^{33}$. Que el movimiento pitagórico gozaba todavía en la Roma del siglo I a.C. de cierta presencia queda patente por un lado en el propio Varrón, quien no ocultaba sus simpatías por esta corriente ${ }^{34}$, y por otro en Ovidio,

27 Véanse, por ejemplo, W. Soltau, «Bot Diodors annalistiche Quelle die Namen der ältesten Volkstribunen?», Philologus, 71, 1912, p. 270; K.J. Beloch, Römische Geschichte, pp. 121 s.

${ }_{28}$ Cf. G. PerL, Kritische Untersuchungen zur Diodors römischer Jahrzählung, Berlin, 1957, pp. 141 ss.; F. JACOBY, FGH, vol. II B, Kommentar, Leiden, 1962, p. 826; R. Werner, Der Beginn der römischen Republik, p. 191; F. CASSOLA, «Diodoro e la storia romana», pp. 743 s.; K.S. SACKs, Diodorus Siculus and the First Century, pp. 65 s.

${ }^{29}$ Cf. S. Mazzarino, Il pensiero storico classico, vol. II, pp. 411 s.; vol. III, pp. 449 s.

30 Cf. Aug., Civ. Dei, 21.8.2. Véanse al respecto L. Ross TAYLor, «Varro's "De gente populi Romani”», $C P h, 29,1934$, p. 221; H. Dalhmann, «M. Terentius Varro», RE, Suppl. VI, 1935, col. 1240.

${ }^{31}$ Cástor, FGH 250 F 15 (= Plut., QRom., 10).

32 H. Peter, Die Quellen Plutarchs in der Biographieen der Römer, Halle, 1865, p. 164; E. GabBA, «Considerazioni sulla tradizione letteraria sulle origini della Repubblica», p. 156 (= Roma arcaica, p. 40).

${ }^{33}$ Cástor, FGH 250 F 5 (= Euseb., Chron., 142 s. K). Véase KuBitscheK, «Kastor von Rhodos», RE, $\mathrm{X}, 1919$, col. $2352 \mathrm{~s}$.

${ }^{34}$ Por ejemplo, Var., L.L., 5.11; 7.17. Sobre el particular, A.D. Nock, «Varro and Orpheus», CR, 43, $1929,61-62$. 
quien en la presentación que hace de la relación Numa-Pitágoras parece seguir una fuente de inspiración no lejana a círculos neopitagóricos ${ }^{35}$.

\section{Diodoro, 8.25-26: Tulo Hostilio y el bellum iustum}

El relato conservado de Diodoro sobre la declaración de guerra del rey Tulo Hostilio a los albanos, que terminará al cabo del tiempo con la victoria romana y la destrucción de Alba, sigue en líneas generales la corriente más común en la analística representada por Livio y Dionisio ${ }^{36}$. Pero dentro de esta uniformidad, es posible diferenciar diversas tendencias. Por un lado se encuentra aquélla personificada en Livio, y que siguen asimismo Floro y $\mathrm{Orosio}^{37}$, que atribuye la responsabilidad del conflicto al rey romano, caracterizado continuamente por su ferocitas ${ }^{38}$. Dionisio y Diodoro, por el contrario, dejan caer toda la culpa sobre los albanos, envidiosos de la potencia romana, y presentan a Tulo, en este episodio, como un buen monarca, respetuoso de las formas legales y de las tradiciones. Por ello, estos autores afirman que Tulo declaró la guerra según la costumbre, de tal manera que el conflicto se adaptaba a los requisitos del bellum iustum, y eximen al rey de Roma de toda culpabilidad. Aquí se observa una segunda diferencia respecto a la tradición seguida por Livio, según la cual habría sido Anco Marcio, sucesor de Tulo en el trono de Roma, quien deseoso de restaurar las buenas relaciones con los dioses quebradas por la actitud de su antecesor, introdujo el ritual de declaración de guerra tomándolo de los equícolas ${ }^{39}$. Dionisio sigue otra versión, pues atribuye a Numa, como creador del colegio de los feciales, la institución del ritual de declaración de guerra ${ }^{40}$, de manera que Tulo no hizo sino aplicar la doctrina introducida poco tiempo antes. Existe por último una tercera variante representada por Cicerón, según el cual habría sido Tulo quien formuló las reglas de declaración de guerra de acuerdo con el ius fetiale ${ }^{41}$.

¿En cuál de las tres versiones se incluye Diodoro? En opinión de F. Taeger, Diodoro se aproxima a Cicerón, y uno y otro derivarían de Polibio ${ }^{42}$. Taeger analiza conjuntamente los dos pasajes de Diodoro, unidos por la idea del bellum pium et iustum, y en efecto no parece que el fragmento 8.26, en el que se describe el ritual, deba ponerse

35 Ovid., Fast., 3.151 ss.; Pont., 3.3 .41 ss., y sobre todo Metam., 15.1 ss. Cf. sobre la cuestión V. BuchHeIt, «Numa-Pythagoras in der Deutung Ovids», Hermes, 121, 1993, 77-99; J.F. Miller, «The Memories of Ovid's Pythagoras», Mnemosyne, 47, 1994, 473-487.

${ }^{36}$ Liv., 1.22.3-7; Dion., 3.2-3.

37 Flor., 1.3.2; Oros., 2.4.9. En la misma línea se sitúa Dion Casio (Zon., 7.6).

38 Así también Serv., Aen., 6.813. Cf. E. Mensching, «Tullus Hostilius, Alba Longa und Cluilius», Philologus, 110, 1966, 102-118; R.M. OgiLvie, A Commentary on Livy. 1-5, pp. 105 s.

39 Liv., 1.32.5-13. También Serv., Aen., 10.14; Auct. vir. ill., 5.4. Esta misma procedencia del ius fetiale, pero sin indicar a cuál de los reyes romanos corresponde su introducción, es mencionada también por el Auct. de praen., 1, y en la inscripción CIL VI.1302, ILLRP 447 (cf. C. Ampolo, «Fertor Resius rex Aequicolus», $P d P, 27,1972,409-412)$. Dionisio por su parte conoce la versión, pero la sitúa en el reinado de Numa (Dion., 2.72.2).

${ }^{40}$ Dion., 2.72.

${ }^{41}$ Cic., Rep., 2.17.31.

42 F. TAeger, Die Archaeologie des Polybios, Stuttgart, 1922, pp. 90 s. 
en relación con Anco Marcio, conforme a la tradición presente en Livio. Sin embargo, en 8.25 Diodoro justifica la actuación de Tulo por el respeto del rey hacia una antigua costumbre, lo que parece indicar que la preocupación por el bellum iustum, con las reglas que lo sancionaban, no surgió entonces, sino que venía de tiempos anteriores. En este sentido, Numa aparece como el referente más adecuado, por lo que el fragmento 8.26 -conocido a través de Tzetzes y no de los Excerpta del emperador Constantino- debería relacionarse con Numa y no con Tulo. Si esta interpretación es correcta, entonces Diodoro coincidiría de nuevo con el mismo filón de tradición que Dionisio, lo que se refuerza con otras dos coincidencias entre ambos relativas al ius fetiale. Cuando Dionisio describe la reforma sacerdotal de Numa, y en concreto sobre los feciales y sus funciones, se refiere por un lado a la obligación de extraditar a los romanos que hubiesen injuriado a una ciudad aliada, y por otro al plazo de treinta días desde el momento en que los feciales presentan reclamación hasta la declaración de guerra $^{43}$. Ambos aspectos aparecen aplicados en el fragmento de Diodoro sobre Tulo.

Por el contrario, el relato de Livio está plagado de contradicciones. En su exposición sobre la reforma de Numa no hay la menor referencia a los feciales. La primera mención de estos sacerdotes figura en la descripción del ritual del tratado de paz entre Tulo Hostilio y los albanos, nec ullius uetustior foederis memoria est ${ }^{44}$. Pero, como hemos visto, el mismo Livio atribuye a Anco Marcio la institución del ritual de declaración de guerra, estipulando un plazo de treinta y tres días entre la rerum repetitio y la indictio belli en vez de los treinta que figura en la versión de Dionisio y Diodoro ${ }^{45}$. En el preámbulo de la exposición dice Livio que Numa instituyó las prácticas religiosas de la paz, con lo cual priva a Tulo de esta innovación sacro-jurídica, pero concede a Anco la creación de las fórmulas de declaración de guerra ${ }^{46}$, cuando lo lógico sería pensar que unas y otras forman parte del mismo cuerpo y por tanto tendrían idéntico origen. Pero hay más. Cuando Livio narra la guerra de Tulo contra los albanos, menciona el envío de delegados romanos a Alba para exigir explicaciones, así como la espera de treinta días para la declaración de las hostilidades ${ }^{47}$. Parece evidente que aunque no se mencione expresamente, tales delegados no pueden ser otros que los feciales, que aplican un procedimiento ya en vigor, en esencia el mismo que aparece en la versión de Dionisio y Diodoro. La contradicción sólo se explica por la utilización, por parte de Livio, de dos fuentes distintas ${ }^{48}$.

La versión implícita en Diodoro y Cicerón se enmarca pues en la línea de tradición más antigua, antes de que la figura de Tulo Hostilio sufriera cierto proceso de degradación con connotaciones de impiedad y violencia. A título de hipótesis, aunque nada se puede afirmar con seguridad, podría suponerse que el cambio de actitud en la historiografía republicana respecto a Tulo fuese consecuencia de determinados hechos, sucedidos en época reciente y protagonizados por supuestos miembros de su misma

${ }^{43}$ Dion., 2.72.5 (extradición), 8 (plazo de 30 días).

${ }^{44}$ Liv., 1.24.5.

${ }^{45}$ Este mismo plazo de 33 días aparece en Serv. auct., Aen., 9.52.

${ }^{46}$ Liv., 1.32.5.

${ }^{47}$ Liv., 1.22.5.

${ }^{48}$ Cf. J. BAYET, «Le rite du fécial et le cornouiller magique», MEFR, 52, 1935, p. 29 (= Croyances et rites dans la Rome antique, Paris, 1971, pp. 9 s.); R.M. OGILvIE, A Commentary on Livy. 1-5, p. 129. 
gens. En la segunda mitad del siglo II a.C. existía una doble tendencia a propósito de los Hostilios, manifestada sobre todo en la actuación de L. Hostilio Mancino durante el asedio a Cartago en el transcurso de la tercera guerra púnica y el subsiguiente enfrentamiento político entre Mancino y Escipión Emiliano ${ }^{49}$. Una de las versiones que circulaban era opuesta a Mancino y favorable a Escipión, según se observa en el relato de Apiano ${ }^{50}$, quien probablemente deriva de Polibio. La segunda presenta por el contrario un panorama más proclive a Hostilio Mancino, y debió gozar de algún éxito a juzgar por los testimonios conocidos ${ }^{51}$.

Esta doble apreciación sobre los Hostilios se refleja en parte también a propósito de la muerte de Tulo. Dionisio menciona dos versiones: una la atribuye a una conspiración urdida por su sucesor Anco, otra al castigo divino mediante un rayo que incendió el palacio y acabó con la vida de toda su familia ${ }^{52}$. Según el historiador de Halicarnaso, la primera de ellas era la más extendida, pero de hecho sólo es recordada por Zonaras ${ }^{53}$, que en última instancia deriva del mismo Dionisio a través de Dion Casio, mientras que la segunda aparece en la mayor parte de las fuentes que refieren el episodio ${ }^{54}$. La causa de la ira de Júpiter, que provocó con el rayo la muerte del rey, era que éste se había descuidado en el cumplimiento de determinado rito, que Tulo pretendía realizar conforme a las prescripciones dejadas por Numa. Pero dentro de esta interpretación general, la actitud que se observa en Livio y en Plutarco es más radical en el juicio sobre Tulo, del que proporcionan una imagen de hombre impío, mientras que Dionisio, aun reconociendo el abandono de ciertas prácticas religiosas por parte del rey, no le llega a calificar en términos tan duros. Se sabe que el analista L. Calpurnio Pisón Frugo, cónsul en el 133, trató sobre la muerte de Tulo, puesto que así lo menciona Plinio ${ }^{55}$, y quizá se situaba en la perspectiva menos desfavorable. Tal actitud tendría su lógica, ya que los Calpurnios se encontraban políticamente muy próximos a los Hostilios, y junto a los Postumios formaban un grupo de oposición a Escipión ${ }^{56}$. Se desconoce si esta versión tiene o no su punto de partida en Pisón Frugo, aunque posiblemente sea más antigua. Tampoco sabemos cómo escenificaba Diodoro el episodio de la muerte de Tulo, pero en cualquier caso parece que su opinión sobre este rey era favorable. Algo similar sucede con Cicerón, quien ofrece una visión positiva de Tulo, pero desconocemos de primera mano cómo describió su

49 Sobre la cuestión, últimamente, L. Cotta Ramosino, Plinio il Vecchio e la tradizione storica di Roma nella Naturalis Historia, Alessandria, 2004, p. 127 ss. Acerca de las relaciones entre Escipión y Mancino, puede verse A.E. Astin, Scipio Aemilianus, Oxford, 1967, pp. 70 s., 99 ss.

${ }^{50}$ App., Afr., 113-114.

${ }^{51}$ Liv., Per., 51; Plin., Nat. hist., 35.22-23; Flor., 1.31.10; Ampel., 32.1.

52 Dion., 3.35.

${ }^{53}$ Zon., 7.6.

${ }^{54}$ Además de Dionisio, esta versión figura en Liv., 1.31.8; Plin., Nat. hist., 2.140; 28.14 (éste invoca como fuente al analista L. Calpurnio Pisón, fr. $10 \mathrm{P}=$ fr. $12 \mathrm{Ch}$; fr. $13 \mathrm{P}=$ fr. $15 \mathrm{Ch}$, respectivamente); App., Reg., 2.2; Plut., Num., 22.11-12; Val. Max., 9.12.1; Auct. vir. ill., 4.4; Aug., Civ. Dei, 3.15.

55 Véase anterior n.48. Sobre estos fragmentos, C. SANTINI, «"Eroi culturali” e annalistica: il caso di Tullo Ostilio», Eutopia, 5, 1996, 85-97 (este autor cree que también Plutarco, cuando habla de la muerte de Tulo, derivaría de Pisón, pero no está claro que así sea).

56 A.E. Astin, Scipio Aemilianus, pp. 316 ss. Véase asimismo D.C. EARL, «Calpurnii Pisones in the Second Century B.C.», Athenaeum, 38, 1960, 283-298. 
muerte debido a una laguna en el texto transmitido de su De re publica. Parece que Cicerón, según se cree a partir de lo que dice Agustín de Hipona, mencionaba también la muerte por el rayo, pero sin connotaciones negativas.

\section{Diodoro, 8.31: Tarquinio Prisco}

El fragmento atribuido a Diodoro acerca de Tarquinio Prisco, a pesar de su brevedad, parece enmarcarse en la corriente más general de la tradición. La presentación que se ofrece de Tarquinio es por completo laudatoria, alabando la educación y el buen carácter de su figura, aspectos que más interesaban al compilador bizantino que transmite la noticia. No debemos por tanto sorprendernos que estén ausentes del texto elementos de la versión canónica que, en el grueso de nuestras fuentes, constituyen el núcleo fundamental del relato, como su origen greco-etrusco y el episodio del águila en el Janículo donde Tanaquil profetiza la futura realeza de su esposo. Pero el texto disponible de Diodoro parece sugerir lo contrario. Según el excerptum, Tarquinio entró en relaciones con Anco cuando alcanzó la mayoría de edad ( $\alpha v \delta \rho \omega \theta \varepsilon i \varsigma)$. Si nos detenemos en este punto, habría entonces que reconocer que Diodoro o bien consideraba a Tarquinio de origen romano y no etrusco, o bien situaba su desplazamiento a Roma cuando todavía era un niño y en consecuencia lo habría hecho acompañado de sus progenitores. Pero ninguna de estas soluciones parece satisfactoria, ya que la versión canónica sobre el origen de Tarquinio estaba ya prefectamente asentada con los primeros historiadores romanos. Era conocida por Polibio, quien sin duda deriva de Fabio Píctor, y probablemente debe reconocerse en un fragmento del poeta Ennio $^{57}$. La alusión a la recién estrenada mayoría de edad debe ser considerada pues como producto de la interpretación y vocación epitomista del compilador bizantino.

Diodoro, quien indudablemente conocía la versión canónica, insiste en la paideia y la areté de Tarquinio, lo que parece esconder la aplicación de un modelo griego. Si esta interpretación es correcta, no sería la única vez que se menciona en las fuentes que Tarquinio recibió una formación griega. Aquí nos encontramos de nuevo con Cicerón y Dionisio de Halicarnaso. Al decir del primero de ellos, Demarato «cum de matre familias Tarquiniensi duo filios procreavisset [Arrunte y Lucumo-Tarquinio], omnibus eos artibus ad Graecorum disciplinam erudiit $t^{58}$. En similares términos se expresa Dionisio, quien a la cultura griega añade también la etrusca como fuentes para la educación de los hijos de Demarato ${ }^{59}$. Quizá también Plinio, quien ofrece sobre Tarquinio la imagen de un personaje de origen y cultura griegas, a la vez que trata de ocultar la componente etrusca de su personalidad, según propuesta de L. Cotta Ramosino ${ }^{60}$. Este vínculo de Tarquinio con el universo helénico se amplía en otras versiones, que le presentan como un auténtico griego. De hecho, para Cicerón parece

57 Pol., 6.11a (cf. F.W. Walbank, A Historical Commentary on Polybius, vol. I, p. 672); Ennio, fr. $147 \mathrm{~V}$ (= Prob., Eccl., 6.31).

58 Cic., Rep., 2.19.34.

59 Dion., 3.46.5.

${ }^{60}$ L. Cotta Ramosino, Plinio il Vecchio e la tradizione storica di Roma nella Naturalis Historia, pp. $142 \mathrm{~s}$. 
como si el nacimiento etrusco de Tarquinio no dejase de ser un mero accidente, ya que era en todo un griego, como lo muestran su educación y cultura y sobre todo su nombre originario, que tuvo que cambiar por el latino de Lucio Tarquinio ${ }^{61}$. Floro, por su parte, va más lejos y califica a este rey de Roma como oriundus Corintho ${ }^{62}$. Finalmente otros autores transmiten una curiosa noticia, según la cual Tarquinio Prisco instituyó el culto a la tríada capitolina por estar iniciado en los misterios de Samotra$\mathrm{cia}^{63}$. El origen de estas últimas versiones parece tener una matriz griega, pero éste ya no es el caso de Diodoro.

Cuando Dionisio se dispone a narrar el origen de Tarquinio, afirma que lo va a

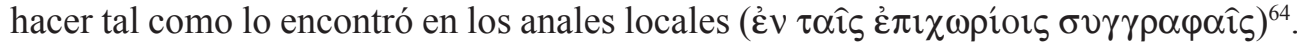
Es más que probable que con tal designación se indique la crónica pontifical. No es ésta la unica ocasión en la que Dionisio invoca los annales maximi en su exposición sobre la Roma primitiva. También al narrar las diferentes versiones sobre el origen de Servio Tulio recurre a esta misma fuente, en concreto cuando relata la maravillosa concepción del monarca a través del fuego del hogar ${ }^{65}$. Pero mayor interés ofrece a nuestros fines una referencia a Rómulo y Remo, conservada en las "tablas sagradas", que contiene diferentes versiones sobre la concepción de los gemelos ${ }^{66}$. Entre ellas menciona una según la cual Rómulo y Remo eran hijos de la hija de Eneas. Esta misma relación parental del fundador de Roma figura en Diodoro, quien invoca a

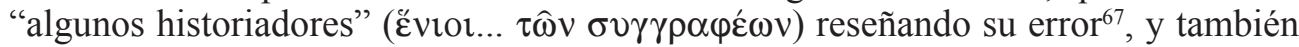
en Nevio y Ennio ${ }^{68}$, lo que dio pie a Th. Mommsen para inclinarse por Fabio Píctor, aproximadamente contemporáneo a los mencionados poetas, como fuente de Diodoro $^{69}$. Sin embargo, y sin negar por completo esta última posibilidad, quizá fuese más oportuno pensar que en última instancia Diodoro deriva de los annales maximi. Se ha podido comprobar que este historiador griego utilizó, directa o indirectamente, la

${ }^{61}$ Cic., Rep., 2.20.35: sic enim suum nomen ex Graeco nomine inflexerat.

62 Flor., 1.5.1 (cf. Val. Max., 3.4.2).

63 Macr., Sat., 3.4.8; Serv. auct., Aen., 2.296. La opinión dominante sostiene que esta tradición procede en última instancia de Varrón (G. WissowA, «Die Überlieferung über dir römischen Penaten», Hermes, 22, 1887, pp. 35 s. [= Gesammelte Abhandlungen, München, 1904, pp. 120 s.]; P. BoYANCÉ, «Sur la théologie de Varron», REA, 57, 1955, pp. 71 s.; B. Combet-FARnoux, Mercure romain, Roma, 1980, p. 210; S.G. Cole, Theoi Megaloi, Leiden, 1984, p. 101). Sin embargo, quizá habría que pensar en una fuente griega, de inspiración órfico-pitagórica, originaria de la Italia meridional (J. MARTíNEZ-PINNA, «La tradición sobre el origen de Tarquinio Prisco», en Atti II Congresso Internazionale Etrusco, Roma, 1989, vol. I pp. 136 ss.; IDEM, Tarquinio Prisco, Madrid, 1996, pp. 17 s.).

64 Dion., 3.46.2.

65 Dion., 4.2.1-2.

66 Dion., 1.73.1-3. Ausente en la reciente recopilación de M. CHASSIGNET, L'annalistique romaine. I. Les annales des pontifes et l'annalistique ancienne, Paris, 1996, esta referencia era sin embargo aceptada como perteneciente a los annales maximi ya por A. ScHWEGLER, Römische Geschichte, vol. I.1, p. 8, n.4, y por O. HiRSCHFELD, «Die kapitolinischen Fasten», Hermes, 9, 1875, p. 107, y más recientemente también por E. GABBA, «Considerazioni sulla tradizione letteraria sulle origini della Repubblica», pp.152 s. (= Roma arcaica, pp. 37 s.), y por B.W. FrIER, Libri Annales Pontificum Maximorum, Roma, 1979, pp. 305 ss.

67 Diod., 7.5.1.

68 Véase Serv. auct., Aen., 1.273.

${ }^{69}$ Th. Mommsen, «Fabius und Diodor», p. 314, n. 2. Según G. VAnotti, «Diodoro epitomato e le origini di Roma», pp. 219 s., se trataría de historiadores influidos por los poetas Nevio y Ennio. 
crónica de los pontífices en su exposición de la dinastía albana ${ }^{70}$, por lo que no tendría nada de extraño que esta influencia se refleje asimismo en otras partes de su relato sobre los reyes de Roma.

\section{Diodoro, 10.1-2: Fin de Servio Tulio}

El fragmento 10.1 de Diodoro se sitúa en los últimos momentos del reinado de Servio Tulio, que culminarán con el asesinato del monarca. La situación se repite, prácticamente en los mismos términos, en los relatos de Livio y de Dionisio. Habiendo urdido una conjura contra su suegro, Tarquinio el Soberbio convoca al Senado para ser proclamado rey. En estas circunstancias, Servio se presenta en la Curia y le reprocha su actitud, ante lo cual Tarquinio invoca el principio dinástico para reclamar su derecho al trono y reprocha a Servio su origen servil. La escena finaliza con la expulsión de Servio de la Curia, siendo arrojado violentamente por las escaleras y muerto por unos servidores de Tarquinio ${ }^{71}$.

Como se sabe, Livio y Dionisio ofrecen interpretaciones diferentes sobre la personalidad política de Servio Tulio ${ }^{72}$. Livio se enmarca en la línea, más antigua, que ve en Servio un monarca proclive a los intereses de la aristocracia, mientras que Dionisio, que se inspira en analistas más recientes, ofrece una imagen de Servio como la de un rex popularis. Esta diferencia se aprecia con absoluta claridad en el procedimiento de entronización de Servio, protagonizado por el Senado en la versión de Livio y por el pueblo en la de Dionisio ${ }^{73}$. Sin embargo, en el episodio que ahora nos ocupa no se trasluce esta oposición, sino que las diferencias entre Livio y Dionisio son sobre todo de carácter narrativo, más conciso en el primero y más desarrollado en el segundo.

A partir de los datos disponibles, no podemos saber sobre cuál de estas dos tendencias de la tradición acerca de Servio Tulio basculaba el relato de Diodoro. Tampoco por la inclinación política del propio historiador, pues aunque sentía especial predisposición hacia César, Diodoro no se deja guiar por sus propias ideas en la reconstrucción del pasado, según ha mostrado D. Ambaglio ${ }^{74}$. A partir del texto de Diodoro sobre la caída del decenvirato, con el acceso de los plebeyos a la magistratura y la definición de los poderes tribunicios como expresión de la máxima potestas

${ }^{70}$ F. CASSOLA, «Le origini di Roma e l'età regia in Diodoro», p. 316. Quizá de forma abusiva, invoca esta misma fuente para Diodoro M. RusConI, «Le notizie romane di Diodoro e gli ‘Annales Maximi'», cit.

71 Liv., 1.48.1-5; Dion., 4.38.2-39.5. Estos dos autores sitúan el asesinato de Servio en un mismo lugar del Esquilino, mientras que Diodoro nada especifica, y aunque del texto conservado pudiera inferirse que Servio fue muerto en las inmediaciones de la Curia, no se debe descartar que tal impresión sea producto del epitomista.

${ }_{72}$ Sobre el particular, me permito remitir a J. MartínEZ-PinNA, «Entre la monarquía y la República según Livio y Dionisio de Halicarnaso», en Hommages P.M. Martin, e.p., con fuentes y bibliografía.

${ }^{73}$ Cf. V. Fromentin, «Servius Tullius sans Fortune? ou la figure du roi Servius Tullius chez Denys d'Halicarnasse», en Pouvoir des hommes, signes des dieux dans le monde antique, Besançon, 2002, pp. 60 ss.

74 D. Ambaglio, La Biblioteca storica di Diodoro Siculo, Como, 1995, pp. 147 ss. Cf. K.S. SACKs, Diodorus Siculus and the First Century, p. 122; M. SARTORI, «Storia, 'utopia' e mito nei primi libri della Bibliotheca Historica di Diodoro Siculo», Athenaeum, 62, 1984, pp. 531 ss. 
en la ciudad ${ }^{75}$, J.-L- Ferrary ha podido demostrar que el historiador siciliano seguía una fuente favorable a la ideología de los populares y posterior a la crisis del año 133 protagonizada por Ti. Graco ${ }^{76}$. ¿Nos puede llevar esto a pensar que sea posible aplicar esta idea a su versión sobre Servio Tulio y que, en consecuencia, utilice el mismo tronco de tradición que Dionisio? Antes de responder afirmativamente, es necesario sin embargo salvar una dificultad.

Según Diodoro, Tarquinio el Soberbio era hijo de Tarquinio Prisco, en lo que coincide con Livio y en última instancia con Fabio Píctor; Dionisio por el contrario prefiere seguir a Calpurnio Pisón, quien establece una relación de abuelo-nieto entre los dos Tarquinios, con una generación intermedia entre ambos, pero sin que ello implique modificar la duración total de la época monárquica ${ }^{77}$. Pero es muy posible que tal elección no le haya sido sugerida a Dionisio por su fuente inmediata, sino que más bien parece producto de su propia investigacion. De ahí también las contradicciones en las que incurre el mismo Dionisio respecto a otros miembros de la familia de los Tarquinios. Así puede deducirse de otra noticia sobre la genealogía de los Tarquinios que figura en los fragmentos de Diodoro, cuando éste define a Bruto, el "padre de la República", como sobrino de Tarquinio el Soberbio, de acuerdo con Livio, Dion Casio y el pseudo-Aurelio Víctor ${ }^{78}$. Indirectamente Diodoro coincide también con Dionisio, según el cual Bruto era nieto de Tarquinio Prisco ${ }^{79}$, al igual que el Soberbio. Pero aquí Dionisio entra en contradicción con su propio esquema genealógico, pues al situar a Bruto en la generación siguiente a la de Tarquinio el Soberbio, se hace necesario alejar más a Bruto de Tarquinio Prisco ${ }^{80}$. En definitiva, Diodoro pudo haber seguido la misma línea de tradición que Dionisio, pero sin modificar el vínculo pa-

75 Diod., 12.25.2.

76 J.-L. Ferrary, «L'archéologie du De re publica (2, 2, 4-37, 63): Cicéron entre Polybe et Platon», $J R S, 74,1984$, pp. 88 s. La oposición Senado-pueblo aparece por vez primera en el texto de Diodoro a propósito de la catástrofe gala (Diod., 14.113.7), dependiente de una fuente tardorrepublicana (de tendencia optimate según F. CASSOLA, «Diodoro e la storia romana», pp. 752 s.).

${ }^{77}$ Fabio, fr. 11a P = fr. 12 Ch (= Dion., 4.6.1; 30.2); Pisón, fr. $15 \mathrm{P}=$ fr. $17 \mathrm{Ch}$ (= Dion., 4.7.5). Sobre la cuestión, P.M. MARTin, «Le souci chronologique dans la tradition sur la généalogie des Tarquins», en AIÔN. Le temps chez les Romains, Paris, 1976, p. 59; L. Bessone, «La gente Tarquinia», RFIC, 110, 1982, pp. 394 ss.; O. De CAZANove, «La chronologie des Bacchiades et celle des rois étrusques de Rome», MEFRA, 100, 1988, pp. 616 ss.

${ }^{78}$ Diod., 10.22; Liv., 1.56 .7 (L. Iunius Brutus, Tarquinia, sorore regis, natus); Auct. vir. ill., 10.1 (Iunius Brutus sorore Tarquinii Superbi genitus); Cas. Dio, 2.11.10; Zon., 7.11.11. Todavía hay una noticia más en Diodoro sobre esta genealogía, ahora referida a Tarquinio Collatino, convertido en "primo" de Tarquinio el Soberbio (Diod., 10.20.1). Se trata de un caso único, puesto que Collatino siempre figura en una generación posterior, es decir nieto (Liv., 1.57.6) o biznieto (Dion., 4.64.3) de Arrunte, hermano de Tarquinio Prisco; incluso el Auct. vir. ill., 9.1, que también representa un caso singular, dice que Collatino era sobrino de Tarquinio el Soberbio. Posiblemente el término que figura en el texto transmitido de Diodoro, $\alpha v \varepsilon \psi$ iós, deba entenderse como "pariente póximo", sin especificar una relación concreta.

${ }^{79}$ Dion., 4.68.1.

80 Véanse T.N. Gantz, «The Tarquin Dinasty», Historia, 24, 1975, p. 543; L. Bessone, «La gente Tarquinia», pp. 396 ss. No es éste el único lapsus que comete Dionisio, quien en otro lugar (5.2.1) dice que Tarquinio el Soberbio era hijo de Tanaquil, y por tanto también del Prisco. 
rental entre los Tarquinios, solución que parece más probable que la otra alternativa representada por el filón de Livio ${ }^{81}$.

El fragmento 10.2 señala el fin del relato sobre el reinado de Servio Tulio, al que Diodoro adjudica una duración de 44 años, cifra que coincide con la proporcionada por Livio y Dionisio ${ }^{82}$. El texto se cierra con una alusión laudatoria a la obra legislativa de Servio y a la virtud de este monarca, aspectos firmemente anclados en la analística más reciente.

Tras este breve recorrido por los escasos fragmentos de Diodoro relativos a los reyes de Roma, pocas conclusiones firmes pueden obtenerse. La exigüidad de la información, unida a las condiciones de transmisión de los fragmentos, no permite ir muy lejos, si bien no se debe renunciar a un pausado avance. Ante todo, Diodoro se incluye plenamente en la línea de la vulgata, salvo quizá leves diferencias cronológicas, sobre todo en lo que se refiere a la fecha de fundación de Roma (751/50 a.C.), que coincide con Polibio y la crónica pontifical ${ }^{83}$. Pero aun manteniéndose en el seno de la "doctrina", Diodoro ofrece mayores discrepancias de detalle con la tradición representada por Livio, y por el contrario notables coincidencias con Dionisio de Halicarnaso, continuanado una relación ya observada en la exposición sobre la disnastía albana ${ }^{84}$, y en parte también con Cicerón. Todo parece indicar que Diodoro y Dionisio utilizaron fuentes bastante próximas, o en cualquier caso se insertan en el mismo tronco de tradición. Resulta difícil aceptar la idea, desarrollada especialmente por F. Taeger, que en su exposición sobre la época monárquica de Roma, Diodoro no utilizaba fuentes locales ${ }^{85}$. No obstante, nada puede afirmarse con absoluta certeza y sólo queda concluir, con K.J. Beloch, «man glaubt gern was man wünscht» ${ }^{86}$.

81 Cf. sin embargo P. FraCCARO, «La storia romana arcaica», RIL, 85, 1952, p. 107 (= Opuscula, Pavia, 1957, vol. I, p. 15). Por su parte, F. SchachermeYr, «L. Tarquinius (Superbus)», RE, IVA, 1932, col. 2381, piensa en Fabio Píctor como fuente de Diodoro, pero sin aportar argumentos precisos. Puede verse asimismo V. GRoH, «La cacciata dei re romani. Analisi letteraria e storica», Athenaeum, 6, 1928, pp. 294 ss., según el cual en el relato sobre la expulsión de Tarquinio el Soberbio, Diodoro se aproxima a Dionisio y en última instancia ambos parecen derivar de Fabio Píctor.

82 Liv., 1.48.8; Dion., 4.40.1.

${ }^{83}$ Acerca de la cronología utilizada por Diodoro para la época monárquica, son fundamentales G. PerL, Kritische Untersuchungen zur Diodors römischer Jahrzählung, pp. 18 ss.; R. WeRnER, Der Beginn der römischen Republik, pp. 166 ss.

${ }^{84}$ F. CASSOLA, «Le origini di Roma e l'età regia in Diodoro», p. 324.

${ }^{85}$ F. TAeger, Die Archaeologie des Polybios, pp. 87 ss. Tal es su conclusión en p. 93: "Die Analyse der einzelnen Fragmente der Königsgeschichte zeigt, daß Diodor keine einheitliche Vorlage benütz hat, sondern da $\beta$ in seiner Darstellung in der Hauptsache zwei Bearbeitungen verwoben sind, von denen die eine durchaus polybianisches, die andere annalistisches Gepräge trägt. Dahinter tritt die Einlage, die auf Poseidonios und seine Schule deutet, zurück" (la relación con Poseidonio parece ser sugerida asimismo por J. HaRmatTa, «Poseidonius über die römische Urgeschichte», $A C D, 7,1971$, pp. 24 s.).

${ }^{86}$ K.J. Beloch, Römische Geschichte, p. 126. 